\title{
Evolución de la oferta de médicos. Chile 1993-2008
}

\author{
OSCAR ROMÁN A.
}

Departamento de Medicina. Campus Centro. Universidad de Chile y Ministerio de Salud. Departamento de Formación y Capacitación.

Recibido el 14 de abril de 2009 aceptado el 11 de noviembre de 2009

Correspondencia: Dr. Oscar Román A Huelén 154 Depto. 2 Providencia Santiago.

\section{Evolution of the availability of physicians in Chile}

There is a significant increase in the physician availability in Chile in the last 15 years, due to the immigration of foreign physicians, but mainly due to the increasing number of graduates from private universities with medical schools. In the last four years, the number of physicians increased from 25.542 to 29.996 and the number of graduates, from 918 to 1.136. These figures show a nearly exponential growth. The number of physicians/number of beneficiaries ratio increased from 1/630 to 1/569 in the last four years, due to the greater increase in the number of physicians than in the number of inhabitants. The future will show a similar trend in this ratio. The specialist/general practitioner ratio remains practically the same, as the number of physicians/beneficiaries ratio in the public system. The oversupply of physicians should alert authorities about the inconvenience of creating new medical schools and to carefully plan the relationship between the offer and demand of medical services.

(Rev Med Chile 2010; 138: 22-28).

Key words: Education, Medical; Public Health; Public Health administration.
E n los últimos 15 años se ha producido un crecimiento importante de las carreras de Medicina debido al aumento del número de Universidades, especialmente privadas. A las 12 carreras de Medicina existentes en el decenio pasado, que podemos considerar tradicionales, se han agregado 14 pertenecientes a otras tantas Universidades privadas, lo que hace un total de 26 carreras $^{1,2}$. Esto determina que el número de médicos que van a estar vigentes en los próximos años va crecer en forma significativa y probablemente superará la necesidad de ellos en relación a la población nacional y a la demanda real de atenciones de salud ${ }^{3-5}$.

Los posibles factores que condicionan la demanda de médicos en la situación actual del país serían la carga demográfica ${ }^{6}$, el aumento de la edad de la población ${ }^{7}$ y el eventual crecimiento de la necesidad de médicos como consecuencia de la reforma de salud ${ }^{8}$.

Por tanto, para una adecuada planificación del recurso médico se hace necesario correlacionar la demanda real de atenciones requeridas por la po- blación con la cantidad de profesionales médicos que la satisfagan. La oferta de atenciones médicas se puede inferir con cierta precisión por el número de médicos vigentes, pero la demanda total de ellas según la población a atender no se conoce con exactitud, sino en base a aproximaciones, utilizando indicadores disponibles para algunas enfermedades y mediante la demanda satisfecha por la oferta. El sistema público posee estadísticas muy aproximadas al respecto ${ }^{4,5,9-11}$.

Las limitaciones para conocer exactamente la demanda obligan a recurrir a indicadores internacionales que relacionan el número de médicos con la población a atender. Los indicadores válidos para nuestra realidad son los de la OMS, que establece 1 médico por cada 600 habitantes y los de la OPS, que para los países de la región, propone 1 médico por cada 1.000 habitantes ${ }^{11-14}$.

Estos indicadores, si bien útiles para establecer metas generales de salud, no consideran algunas limitantes, como la distribución regional, la relación urbano-rural y la del sector público con el privado. Además, las estadísticas de atención de salud no 
miden las necesidades reales y no incluyen la demanda insatisfecha ni los tiempos de espera, por lo que es difícil correlacionar la oferta de médicos con los requerimientos de atención del país s,15-17. $^{\text {. }}$

Otro problema en la planificación del recurso médico se refiere a la relación entre médicos generales y especialistas. En el sistema actual, se prioriza las atenciones secundaria y terciaria, que requieren especialistas, en desmedro de la atención primaria, que sirven los médicos generales y que constituye el pilar fundamental de la reforma de

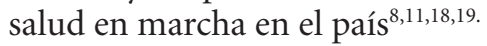

Esta investigación tiene como objetivo establecer los cambios en la oferta de médicos en los dos últimos decenios en el país, con el objeto de cuantificarlos en su proyección futura.

\section{Metodología}

Como no se dispone de una estadística anual del número real de médicos vigentes en el país, éste se obtuvo, desde 1993 a 2003, de los datos comunicados por $\mathrm{Goic}^{3,19}$ en base a la estimación del número de titulados anualmente por las Escuelas de Medicina, sumados a los graduados en el extranjero y habilitados por ley para ejercer en el país. Para cada año, Goic calculó tasas estimadas de mortalidad y de retiro. Posteriormente, desde el año $2004 \mathrm{al} 2008$ las cifras se obtuvieron de las estadísticas reales elaboradas por el Departamento de Recursos Humanos del Ministerio de Salud, en base a un verdadero trabajo de inteligencia computacional que recogió datos de los registros del Consejo de Educación Superior respecto a las promociones de las carreras de Medicina de todas las Universidades, de los inscritos en el Colegio Médico de Chile, Fonasa, Servicio de Impuestos Internos (boletas y declaraciones) y Ministerio de Relaciones Exteriores y Universidad de Chile (reválida) para los inmigrantes ${ }^{4,20,21}$. Las estimaciones a futuro 2010, 2012 se obtuvieron aplicando una regresión lineal de las matrículas de todas las carreras de Medicina de las Universidades nacionales, modificadas por una tasa de abandono estimada en $3,5 \%{ }^{21}$.

Los médicos generales se han obtenido del registro del Departamento de Atención Primaria del Ministerio de Salud ${ }^{18}$ y los especialistas, de la información proporcionada por CONACEM y por las Escuelas de Graduados de las Carreras de Me- dicina $^{22,23}$. Los datos demográficos se obtuvieron del Instituto Nacional de Estadística ${ }^{6}$.

\section{Resultados}

1. Número de médicos. Desde el año 1993, en que el número estimado por Goic era de $15.451^{3}$, éste aumentó en forma muy moderada hasta 1998, año en que alcanza a 16.760 (Tabla 1). Luego, en los cortes sucesivos en los años 2004 y 2008, se incrementó significativamente, llegando a 29.996 en el último año.

Las estimaciones del número de médicos, basadas principalmente en los egresos de las escuelas de medicina, han resultado inferiores al número real en todos los años, con excepción de la de 1998, que lo sobreestimó en un porcentaje bajo $(6 \%)$. Ello se ha debido, probablemente en parte, a la inmigración de médicos extranjeros, que han aumentado desde 711 en 1990 a 3.239 en $2004^{3,4}$. En total la estimación a futuro, para los años 2010 y 2012, calculada en base a las matrículas de todas las carreras actuales de medicina ${ }^{21}$, alcanza a 34.345 y 37.197 médicos, respectivamente (Tabla 1).

La relación número de médicos por población total del país, de acuerdo a los resultados de los censos poblacionales de 1992 y 2002 del INE $^{6}$ y de sus estimaciones hasta el 2012, ha variado desde 1 médico por 915 habitantes en 1994 a 1/894 en $1998,1 / 630$ en el 2004 y 1/569 en el 2008. La estimación del incremento de médicos y de población haría llegar este indicador a 1/533 en el 2010 y a 1/498 en el 2012 (Tabla 2).

$\mathrm{Al}$ correlacionar los valores de nuestro indicador No médicos/población, con el referente teórico $1 / 600$, que la OMS postula como adecuado ${ }^{11,16}$, se observa que se sitúa por debajo entre 1994 y 1998,

Tabla 1. Total de médicos vigentes en Chile 1993-2008 y las estimaciones correspondientes hasta 2012

\begin{tabular}{|ccc|}
\hline Año & Número real & Número estimado \\
\hline 1993 & 15.451 & 15.147 \\
1998 & 16.760 & 17.941 \\
\hline 2004 & 25.542 & 20.610 \\
2008 & 29.996 & 24.449 \\
2010 & --- & 34.345 \\
\hline 2012 & --- & 37.197 \\
\hline
\end{tabular}


Tabla 2. Relación entre número de médicos y población total. Chile 1994-2010

\begin{tabular}{|cccc|}
\hline Año & $N^{\circ}$ médicos & Población & Médico/habitantes \\
\hline 1994 & 15.451 & 14.151 .708 & $1 / 915$ \\
\hline 1998 & 16.760 & 14.996 .647 & $1 / 894$ \\
\hline 2004 & 25.542 & 16.093 .378 & $1 / 630$ \\
\hline 2008 & 29.996 & 16.611 .101 & $1 / 553$ \\
\hline 2010 & $34.345^{*}$ & $17.094 .270^{*}$ & $1 / 533^{*}$ \\
\hline 2012 & $37.197^{*}$ & $17.402 .630^{*}$ & $1 / 498^{*}$ \\
\hline
\end{tabular}

*: Cifras estimadas.

lo iguala en el 2004 y lo sobrepasa en el 2008 (Tabla 2). Las extrapolaciones hasta el 2012 mantienen la tendencia al incremento.

De los resultados obtenidos se desprende que el porcentaje de aumento del número de médicos es significativamente mayor que el del crecimiento vegetativo de la población (Figura 1).

2. Médicos egresados de las universidades nacionales. Los datos proporcionados por las Universidades y el Consejo de Educación Superior $^{2,21}$ revelan un aumento decenal persistente pero moderado del número de egresados entre 1951 y 1991, Luego aumenta en forma notoria hasta el 2004, concordando con el incremento de los profesionales egresados de las carreras de medicina emergentes, la mayoría correspondiendo a las Universidades privadas ${ }^{24}$. Después hay un período de estabilización hasta el año 2007, para nuevamente crecer en el año 2008, alcanzando una cifra superior a los mil egresados por año (Tabla 3 y Figura 2). Por último, las cifras estimadas a futuro, basadas en el número de matrículas de todas las carreras de medicina, clásicas y emergentes, alcanzan a 1.366 profesionales en el año 2010 y 1.426 en el 2012. Los últimos incrementos reales y estimados, se deben a la incorporación de los profesionales egresado de las nuevas Universidades privadas $^{24,25}$.

3. Relación Médicos generales y especialistas. En la Tabla 4 se muestra la evolución de la relación entre generalistas y especialistas desde 2004 a 2008. La proporción de médicos generales se redujo desde el 19 al 15\% en los cuatro últimos años. Además, los especialistas certificados por CONACEM aumentaron en este período, pero su destino laboral hacia el sector público prácticamente no cambió, en tanto que aumentó hacia el sector privado ${ }^{18,22}$.

Por otro lado, la distribución regional de los médicos en la atención de la población beneficiaria

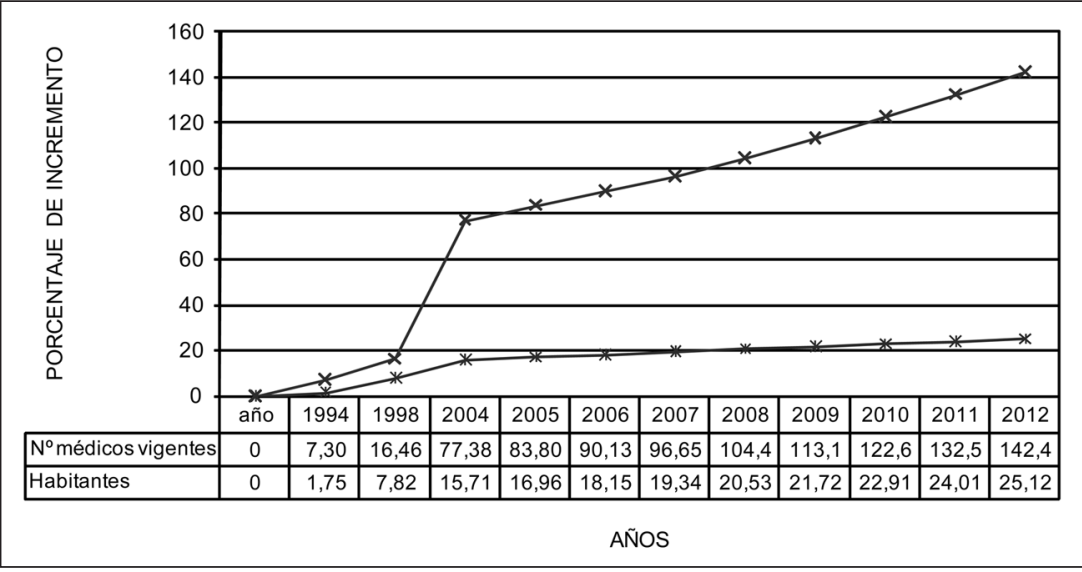

Figura 1. Comparación entre el porcentaje de aumento de médicos y de la población total. Chile 1994-2008. 
FONASA, expresada como tasa por cada 10.000 beneficiarios, no ha variado significativamente en relación a lo comunicado por nuestro grupo en el año 2004. La Figura 3 muestra similar distribución regional, pero la tasa de especialistas aumentó de 4,9 a 6,3 y la de médicos generales se redujo

Tabla 3. Médicos egresados de las universidades nacionales. Número real observado y estimación a futuro chile 19512012

\begin{tabular}{|cccc|}
\hline Año & $\begin{array}{c}\mathbf{N}^{\mathbf{0}} \\
\text { egresados }\end{array}$ & Año & $\begin{array}{c}\mathbf{N}^{\mathbf{0}} \\
\text { egresados }\end{array}$ \\
\hline 1951 & 120 & 2005 & 925 \\
1961 & 250 & 2006 & 912 \\
1971 & 350 & 2007 & 938 \\
1981 & 500 & 2008 & 1.138 \\
1991 & 530 & 2009 & 1.245 \\
2001 & 615 & 2010 & 1.366 \\
\hline 2002 & 738 & 2011 & 1.426 \\
\hline 2003 & 718 & 2012 & 1.426 \\
\hline 2004 & 918 & & \\
\hline
\end{tabular}

Desde 2008 cifras estimadas de acuerdo a matrículas y abandonos calculados en 3,6\% anual. levemente, de 2,3 a 1,9/10.000 beneficiarios ${ }^{4}$. El promedio nacional se mantuvo próximo a los 8,2 médicos por 10.000 beneficiarios $^{18}$.

En los últimos años el número de médicos vigentes en el país se ha podido determinar con mayor precisión gracias a que se ha perfeccionado el acceso a fuentes de información confiables, que ya hemos mencionado ${ }^{4,21,22}$. Ello ha permitido establecer que desde el año 2004 se ha producido un aumento real del número de profesionales, que ha superado al estimado por estudios previos ${ }^{2,3}$. Se ha reconocido que ello se ha debido al aumento de las carreras creadas por las Universidades privadas emergentes y a la inmigración de médicos, chilenos y extranjeros, titulados en Universidades del exterior $^{1,4,24}$. Los inmigrantes también han aumentado significativamente desde 1993, alcanzando cifras cercanas al 10\% del total de médicos registra$\operatorname{dos}^{3,4}$. Ambos factores han determinado que los incrementos reales de médicos hayan superado las estimaciones desde los años 2004 a 20083,4.

Sin embargo, el conocimiento del número de médicos no tiene valor si no se correlaciona con la población total del país a la que debe atender. $\mathrm{La}$ Tabla 2 y la Figura 1 muestran que el crecimiento porcentual del número de médicos es superior al de la población del país. Esta observación con-

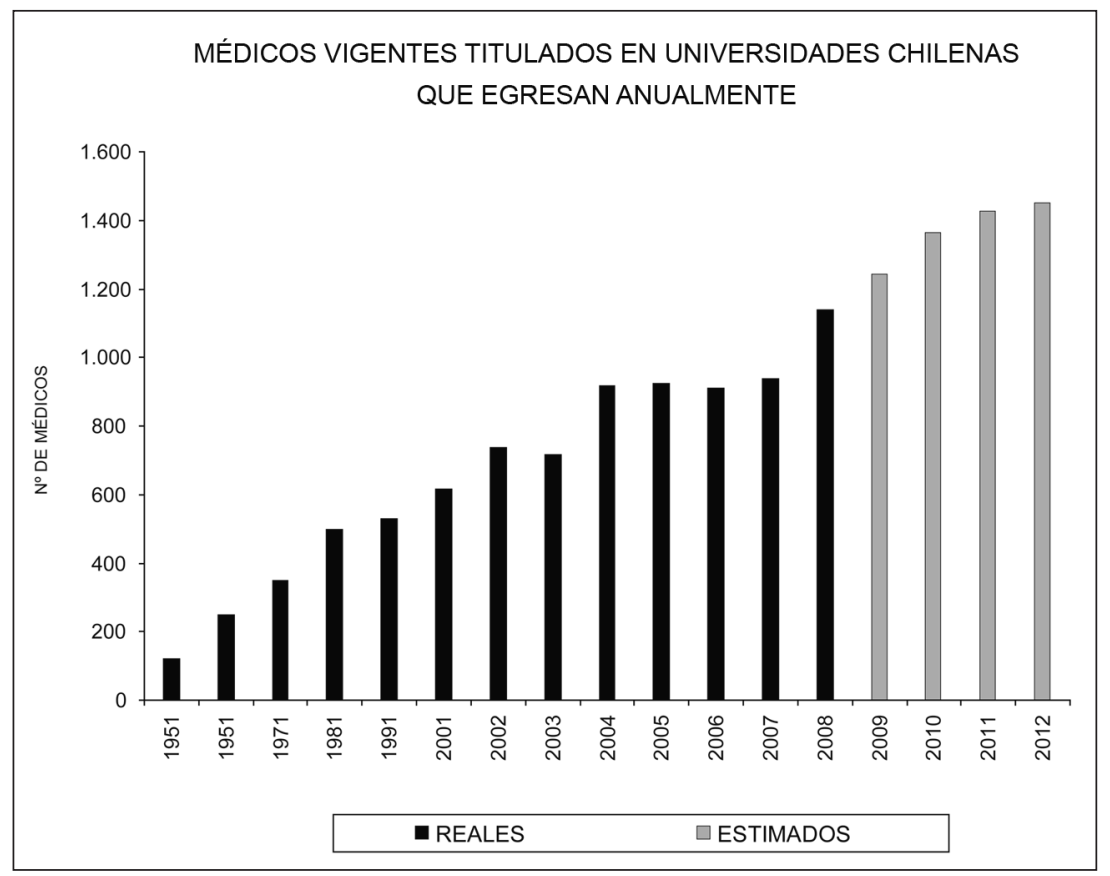

Figura 2. Médicos titulados anualmente en universidades chilenas desde 1951 a 2008. Número estimado hasta 2012. 
Tabla 4. Relación entre número de médicos generales y especialistas en el sistema de servicios de salud. Chile 2004-2008

\begin{tabular}{|c|c|c|c|c|}
\hline \multirow[b]{2}{*}{ Tipo de médicos } & \multicolumn{2}{|c|}{2004} & \multicolumn{2}{|c|}{2008} \\
\hline & $\mathbf{N}^{\circ}$ & $\%$ & $\mathbf{N}^{\circ}$ & $\%$ \\
\hline Total médicos registrados & 8.519 & & 9.581 & \\
\hline Médicos generales & 1.595 & 19 & 1.478 & 15 \\
\hline Médicos especialistas & 6.924 & 81 & 8.103 & 85 \\
\hline Especialistas certificados* & 2006 & & 2008 & \\
\hline Contratados SNSS & 4.088 & & 3.981 & \\
\hline Sólo en sector privado & 5.487 & & 6.150 & \\
\hline
\end{tabular}

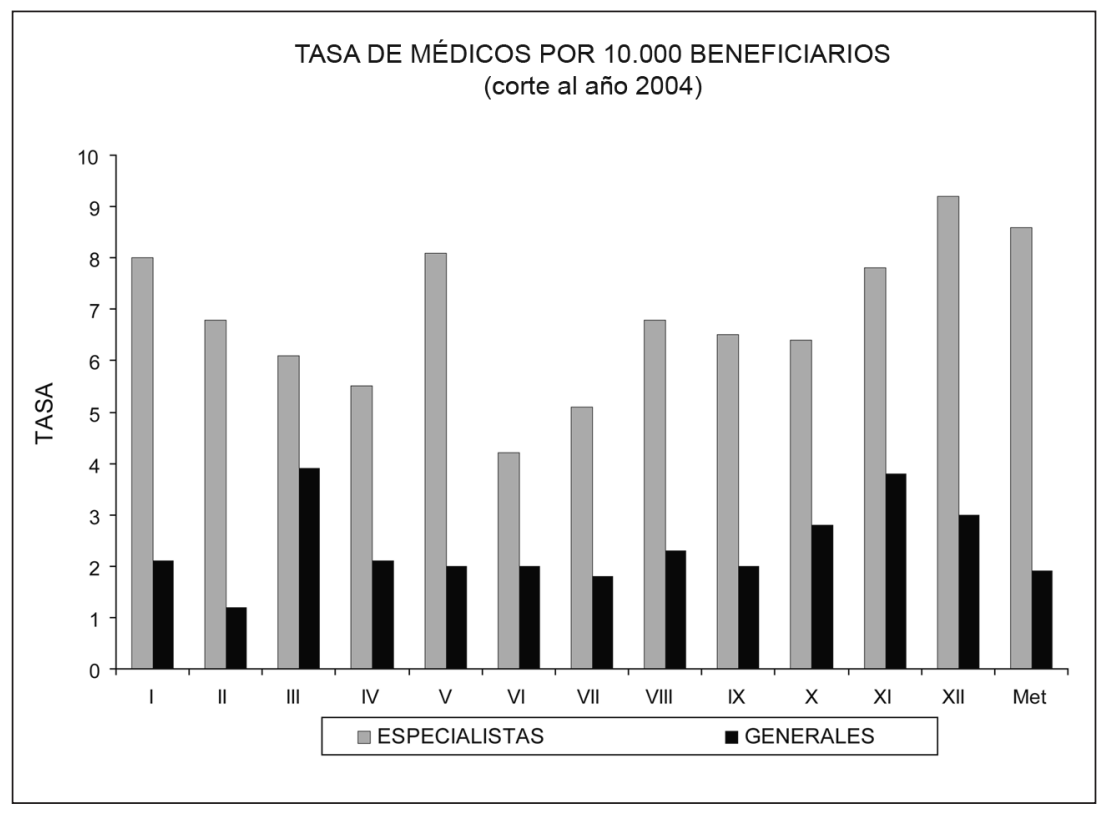

Figura 3. Tasa de médicos generales y especialistas por 10.000 beneficiarios Fonasa y su distribución regional. Corte 2008. cuerda con la de otros países. Así, en Canadá el crecimiento de la población ha aumentado en $72 \%$ desde 1970 a 2000 , en tanto que el número de médicos lo ha hecho en $116 \% 7$. En Perú se ha observado un hecho similar, puesto que desde 1985 hasta 1996 la tasa de crecimiento geométrico de los médicos ha sido de $4 \%$ anual, en tanto que la del demográfico ha sido de $1,7 \%$ anual ${ }^{16}$.

Respecto a la influencia de la edad de la población en el requerimiento de médicos, que podría determinar un aumento de éste, el estudio de Denton y $\mathrm{col}^{7}$ ha demostrado que el crecimiento demográfico total ha sido más importante que el de la edad en la necesidad de médicos?
La evolución del número de médicos no tendría significación si no se lo analiza en función de la demanda de atenciones de salud, por cuanto si la relación inicial hubiera sido deficitaria en forma importante, el incremento de profesionales pudo haber sido justificado. Como la demanda real no se conoce con exactitud, puede ser estimada utilizando indicadores internacionales que relacionan el número de médicos con la población a atender. Para ello hemos escogido la relación 1/600, de acuerdo a la $\mathrm{OMS}^{11}$ como un referente para determinar en forma aproximada y global la evolución de la relación oferta y demanda de médicos en el país. Este indicador ha crecido en forma importan- 
te, desde cifras inferiores al referente internacional en el año 1994 a superiores en el año 2008 y en las estimaciones hasta el 2012 (Tabla 2).

Si bien el aumento de la relación médicos/ población puede ser considerado favorable, debe ser evaluado en función de las necesidades de atención de la población. Si bien éstas no se conocen con exactitud, es preciso señalar algunos hechos importantes que pueden hacerlas variar a futuro. Uno de ellos es el envejecimiento poblacional, que significa mayor carga de enfermedades, aunque haya sido subvalorada por el estudio de Denton y col., que ya hemos comentado ${ }^{7}$. Otro factor es el aumento de la necesidad de especialistas y médicos generales que requiere la Reforma de Salud para la implementación de una atención garantizada de 56 patologías ${ }^{5,17}$. El Ministerio de Salud ha reconocido una brecha en la necesidad de horas médicas para satisfacer esas patologías GES (garantías explícitas de salud) 4,5,10,17.

Por otro lado, también existe carencia de médicos en función de las necesidades del sistema público de salud. En efecto, la tasa global de médicos para la población beneficiaria FONASA es de 8,2/10.000 personas, inferior a la recomendada por la OPS para los países de la región, que es de 10 médicos $/ 10.000^{14}$.

Respecto al aumento de las carreras de medicina, es preciso señalar que de las 26 carreras de medicina hasta el 2008, 10 iniciaron su funcionamiento desde el 2001 y ha habido egresados de la la casi totalidad de ellas. En consecuencia, existe un aumento creciente y significativo en la producción de médicos por las universidades nacionales, como se observa en la Figura 2 y Tabla 3 ).

El incremento de egresados, tanto de Universidades públicas como privadas, obliga a realizar una mirada atenta a la calidad de la formación ${ }^{25,26,27}$. Por ello adquiere relevancia el proceso de acreditación de las carreras que se ha cumplido en 7 de las 14 carreras emergentes ${ }^{2,21,26}$.

Además, la muy posible sobreoferta de médicos en el futuro próximo debe hacer reflexionar sobre la inconveniencia de la creación de nuevas carreras de medicina y de la necesidad que exista una planificación conjunta de la formación médica y las necesidades de la población entre el Ministerio de Salud y el Consejo de Educación Superior ${ }^{12,21}$. En este proceso debería adquirir relevancia la Comisión Nacional Docente-Asistencial ${ }^{2,27,29}$.

Por otra parte, el crecimiento casi exponencial de la oferta de médicos en el país es preocupante porque hace plantear la interrogante si se ha llegado a una saturación del mercado de la salud y por tanto a la posibilidad de cesantía profesional, como se ha señalado en otros países ${ }^{4,16}$. Sin embargo, es posible que no se haya alcanzado en el país un punto de saturación de la oferta, por cuanto desconocemos la realidad del sector privado, que recibe más de la mitad de la producción de médicos, especialmente en calidad de especialistas ${ }^{4,18,22}$. El único indicador de saturación del sector privado sería un aumento de postulantes a las becas y cargos del sector público en la atención primaria de salud, lo que ha comenzado a ser observado por el Ministerio de Salud ${ }^{18,26}$.

Además es necesario reconocer que las cifras comunicadas son eminentemente de tipo cuantitativo, y no informan sobre la calidad del proceso docente y de su correlación con las necesidades asistenciales ${ }^{2,20,24}$. Tal vez el Examen Médico Nacional podría ser una cierta aproximación al problema de la calidad de los egresados de las distintas Universidades del país, pero es evidente que no mide habilidades ni criterios clínicos necesarios, tanto para los egresados nacionales como extranjeros ${ }^{28}$.

En cuanto a la proporción de médicos generales y especialistas, ella se ha mantenido similar a la de 4 años atrás, lo que revela que las medidas tomadas por el Ministerio de Salud y las Municipalidades para reducir la brecha en la atención primaria, estimada en cerca de 1.200 profesionales, no han tenido un resultado efectivo. La relación en el sistema público se mantiene aproximadamente en un 65 versus $35 \% \%^{4,14,18}$.

Con respecto al sector privado, hasta el año 2008, CONACEM ha certificado 6.150 especialistas, en tanto que los correspondientes al sector público llegan sólo a 3.981 ${ }^{4,23}$. El predominio de especialistas en el sector privado sugiere que en éste los médicos tienen mejores expectativas económicas y de progreso tecnológico. Además, en el sector público no se ha producido un aumento de los cargos médicos en el sistema, por lo que, frente a la ausencia de una normativa al respecto, se ha debido recurrir al sistema de contratos a honorarios para paliar la brecha de especialistas, que se ha hecho crítica en lo que concierne al sistema GES o de garantías en salud ${ }^{5,26}$.

En resumen, conocida la eventual sobreoferta de médicos producida hasta el presente, el escaso 
aumento de cargos en los servicios de salud y municipalidades, y el marcado predominio de especialistas versus generalistas, se hace necesario planificar en mejor forma la producción de profesionales en relación a las necesidades de salud de la población.

Agradecimientos: A Luis Echavarría, Ingeniero Comercial USACH y Magíster en Administración hospitalaria, U. Autónoma de Chile y a Fabiola Galleguillos, Administradora Pública.

\section{Referencias}

1. Cruz Coke R. Las Universidades chilenas 1981-2004. Rev Med Chile 2004; 132: 43-8.

2. Armas Merino R, Goic A. Informe sobre la situación actual de la Educación Médica en Chile. Comité de Educación Superior. Academia de Medicina. 2008 Publ. Academia de Medicina.

3. Goic A. Disponibilidad de médicos en Chile y su proyección a mediano plazo. Cinco años después. Rev Med Chile 1999; 127: 1183-88.

4. Román O, Acuña M, Señoret M. Disponibilidad de médicos en Chile al año 2004. Rev Med Chile 2006; 134: 1057-64.

5. Ministerio de Salud. Secretaría Técnica del Régimen de Garantías. Capacidad instalada del sector público de Salud. Informe país. 2006.

6. Instituto Nacional de Estadísticas. Informe censal de población del país y sus estimaciones anuales. (2008).

7. Denton FT, Gafni A, Spencer B G. Requirements for physicians in 2030: Why population aging matters less than you may think. CMAJ 2003; 168: 1545-47.

8. Diario Oficial. Ley 19.937. Modifica el DL. 2.763 de 1979 con la finalidad de establecer una nueva concepción de la autoridad sanitaria, distintas modalidades de gestión y fortalecer la participación ciudadana. Febrero 2004.

9. Departamento de Estadística e Información de Salud (DEIS). Ministerio de Salud. Algunos indicadores de salud. http//www.minsal.cl

10. Ministerio de Salud. Verificación del costo esperado por beneficiario del conjunto priorizado de problemas de salud con garantías explícitas. Informe Integrado. Enero 2007.

11. Organización Mundial de la Salud. Informe sobre la salud en el mundo. Colaboremos por la salud. 2006. www. who.int/whr/2006.

12. Hall T. Taller "modelos de simulación y planificación en Recursos Humanos de Salud" Ministerio de Salud. Marzo 2004.

13. Bastías G, Marshall G, Zúñiga D, Mena B. Número de médicos en Chile: estimaciones, proyecciones y comparación internacional. Rev Med Chile 2000; 128: 1167-76.

14. Organización Panamericana de la Salud. Foro debate ¿Cuántos médicos y enfermeras necesita un país? 2007 http//healthworkers.paho.org.

15. Medina E, Kaempffer AM. Necesidad de médicos en Chile. Rev Med Chile 1998; 116: 389-94.

16. Carrasco V, Lozano E Velásquez E. Análisis actual y prospectivo de la oferta y demanda de médicos en el Perú. Acta Med Per 2008; 25: 22-29.

17. Diario Oficial. Ley 19.996. Del régimen de garantías en salud. Septiembre 2004.

18. Román $\mathrm{O}$, Pineda $\mathrm{S}$, Señoret M. Perfil y número de médicos generales que requiere el país. Rev Med Chile 2007; 135: 1209-15.

19. Goic A. Disponibilidad de médicos en Chile y su proyección a mediano plazo. Rev Med Chile 1994; 122: 141-53.

20. Universidad de Chile. Decreto Exento 6895. Reglamento sobre reconocimiento, revalidación y convalidación de títulos profesionales y grados académicos obtenidos en el extranjero. Octubre 1993.

21. Consejo Superior de Educación. Índices 2007 de carreras profesionales y técnicas.

22. Román O, Señoret M. Estado actual de las especialidades médicas en Chile: realidad en el sistema público no municipalizado. Rev Med Chile 2008; 136: 99-106.

23. Comisión Nacional de Certificación de Especialidades Médicas (CONACEM). Registro de Certificación de Especialidades Médicas. Vida Médica 2004; 56: 18-19.

24. Medina E, Kaempffer R. Medicina y otras carreras de la salud en Chile. Un análisis preliminar. Rev Med Chile 2007; 135: 1346-54.

25. Goic A. Proliferación de escuelas de medicina en Latinoamérica: sus causas y consecuencias. Rev Med Chile 2002; 130: 917-24.

26. Román O. Las nuevas escuelas de medicina en el panorama médico actual. Rev Med Chile. 2009; 137: 1093-98.

27. Academia Chilena de Medicina. Control de calidad en Medicina. Posición de la Academia Chilena de Medicina. Rev Med Chile 2001; 129: 819-21.

28. Comisión del Examen Médico Nacional. Propuesta preliminar del Examen Médico Nacional. Abril 2002.

29. Ministerio de Salud. Normativa Administrativa No 18. Asignación y uso de los campos de formación profesional y técnica en el Sistema Nacional de Servicios de Salud. Octubre 2007. 